\title{
Functional aspects of peritoneal macrophages in endometriosis of women
}

\author{
G. A. J. Dunselman, M. G. R. Hendrix*, P. X. J. M. Bouckaert and \\ J. L. H. Evers \\ Departments of Obstetrics and Gynaecology and of *Medical Microbiology, Maastricht University \\ Hospital and the University of Limburg, P. O. Box 1918, 6201 BX Maastricht, The Netherlands
}

\begin{abstract}
Summary. Peritoneal fluid was collected in the periovulatory phase of the cycle from 25 women undergoing laparoscopy. Endometriosis was diagnosed in 13 patients (AFS score $1, N=9$; AFS score $2, N=4$ ) and 12 patients without endometriosis served as controls. In endometriosis patients the total peritoneal fluid cell number and cell concentration was significantly higher than in controls, indicating peritoneal irritation by endometrial implants. Peritoneal fluid macrophages in patients with endometriosis showed significantly increased erythrophagocytosis and lower chemiluminescence than in controls, suggesting an advanced differentiation of the macrophages in endometriosis patients. The macrophages in this stage of differentiation may interfere with gametes and embryos and thus contribute to endometriosis-associated subfertility.
\end{abstract}

Keywords: endometriosis; peritoneal macrophages; phagocytosis; chemiluminescence; women

\section{Introduction}

Although the association between endometriosis and infertility is accepted, its nature is far from being understood. In recent years peritoneal fluid and its cellular constituents have received considerable attention in the process of elucidating the causes of infertility; peritoneal macrophages are particularly supposed to play an important role in endometriosis-related subfertility (Haney et al., 1981; Badawy et al., 1981; Halme et al., 1982; Olive et al., 1985). Phagocytosis of spermatozoa by peritoneal macrophages has been reported to be increased in endometriosis patients (Muscato et al., 1982).

In this study we determined the cell number of the peritoneal fluid and functional aspects of peritoneal macrophages, erythrophagocytosis and chemiluminescence, reflecting the level of differentiation of the macrophages (Cohn, 1978).

\section{Materials and Methods}

Patients, fluid collection and analysis. Peritoneal fluid was collected in the periovulatory phase of the cycle from 25 women undergoing laparoscopy. The indication for laparoscopy was infertility $(\mathrm{N}=16)$, abdominal pain $(\mathrm{N}=3$ ) and sterilization $(\mathrm{N}=6)$. All patients had regular cycles varying in duration between 23 and 35 days. They had not used oral contraceptives or ovulation-inducing drugs or an IUCD for at least 3 months before the laparoscopy. Patients with occluded Fallopian tubes were excluded.

At laparoscopy the presence or absence of endometriosis was recorded. Endometriosis was classified according to the classification of the American Fertility Society (1979).

Peritoneal fluid was collected under direct vision from the pouch of Douglas and the vesicouterine space as described by Bouckaert et al. (1986). Care was taken to collect all peritoneal fluid. The peritoneal fluid was collected in glass tubes, $1.5 \mathrm{mg}$ sodium ethylenediaminetetra-acetic acid (EDTA) $/ \mathrm{ml}$ were added as anticoagulant and the tubes were stored on ice.

Before induction of anaesthesia a 5 - $\mathrm{ml}$ blood sample was withdrawn, allowed to clot and centrifuged. The supernatant serum was stored at $-70^{\circ} \mathrm{C}$. 
The periovulatory phase of the cycle in which the laparoscopy was to be performed was determined using knowledge of the length of the previous cycles and the first day of the last menstrual period. The concentrations of oestradiol-17 $\beta$ and progesterone measured in serum and peritoneal fluid allowed further subdivision of the periovulatory phase into the follicular and luteal phases (Bouckaert et al., 1986). To identify patients with subclinical inflammatory pelvic disease, C-reactive protein was measured in peritoneal fluid and serum, using single radial immunodiffusion (Mancini et al., 1965).

The peritoneal fluid was centrifuged at $350 \mathrm{~g}$ at $4^{\circ} \mathrm{C}$ for $10 \mathrm{~min}$ to harvest the peritoneal cells. The supernatants were collected and stored at $-70^{\circ} \mathrm{C}$. The pellet was resuspended in Hank's balanced salt solution. Peritoneal cells were counted and the viability determined by trypan blue exclusion. Cells were identified after May-GruenewaldGiemsa fixation and staining from morphological criteria such as polymorphous nuclear cells (PMN), lymphocytes or macrophages.

Erythrophagocytosis and chemiluminescence. Fc-mediated erythrophagocytosis (sheep red blood cell phagocytosis) was measured as described previously (Hendrix et al., 1986). Briefly, peritoneal cell monolayers were prepared in $16-\mathrm{mm}$ wells on 13-mm round glass coverslips by incubating for $1 \mathrm{~h}$ at $37^{\circ} \mathrm{C}$. After adherence, antibody-coated sheep erythrocytes were added to the monolayers and incubated for $1 \mathrm{~h}$ at $37^{\circ} \mathrm{C}$. Non-adherent erythrocytes were subsequently removed by vigorous washing with phosphate-buffered saline (PBS). Each coverslip was then incubated for $10 \mathrm{~min}$ in a $0.85 \%(\mathrm{w} / \mathrm{v})$ ammonium chloride (Merck) solution to lyse non-internalized erythrocytes. After fixation the numbers of macrophages containing no erythrocytes were counted by phase-contrast microscopy. At least 200 cells were counted for each experiment. The results are expressed as the percentage of peritoneal cells containing at least one erythrocyte. Chemiluminescence was measured with a lumino-aggregometer, using opsonized zymosan as stimulating agent, as previously described (Hendrix et al., 1986). Opsonized zymosan was prepared by boiling $8 \mathrm{~g}$ zymosan (Sigma Chemical Co., St Louis, MO, U.S.A.) for $30 \mathrm{~min}$ in $2 \mathrm{ml} \mathrm{PBS}$, centrifuging at $350 \mathrm{~g}$ for $10 \mathrm{~min}$ and resuspending in $200 \mu \mathrm{l}$ normal human AB serum. After incubation for $30 \mathrm{~min}$ at $37^{\circ} \mathrm{C}$ the solution was centrifuged again and resuspended in HBSS to a final concentration of $4 \mathrm{mg} / \mathrm{ml}$. Chemiluminescence was evaluated by introducing $0 \cdot 1 \mathrm{ml}$ of a cell suspension containing $2 \times 10^{6}$ peritoneal cells, $0 \cdot 1 \mathrm{ml} \mathrm{HBSS}+0 \cdot 1 \%(\mathrm{w} / \mathrm{v})$ gelatin (Difco, Detroit, Michigan, U.S.A.), 0.1 ml luminol $\left(10^{-4} \mathrm{M}\right)$ (Lumac, Landgraaf, The Netherlands) and $0.1 \mathrm{ml} 50 \%$ human AB serum in a silicon-coated glass vial, and placing it in the Chronolog. When the background light emission became constant, $0 \cdot 1 \mathrm{ml}$ of the appropriate zymosan suspension was added and the photo-emission was scored continuously during the first 10 min of the reaction. The chemiluminescence response was recorded in duplicate for each experiment. Results are shown as a relative value (units) for the light emission calculated from the total area under the curve during the first 10 min of the chemiluminescence response.

Analyses. Comparisons were made between patients with endometriosis and patients without the disease for the following values: total peritoneal cell number, cell number per $\mathrm{ml}$ peritoneal fluid, percentages of macrophages in the differential counts of peritoneal cells, viability of the macrophages, sheep RBC phagocytosis and chemiluminescence.

Statistical analysis was performed using the Wilcoxon rank sum test for non-paired samples.

Table 1. Total numbers and concentrations of peritoneal cells

\begin{tabular}{|c|c|c|c|c|c|c|}
\hline & \multicolumn{3}{|c|}{ Endometriosis samples } & \multicolumn{3}{|c|}{ Control samples } \\
\hline & $n$ & Median & Range & $n$ & Median & Range \\
\hline Cell no. $\times 10^{-7}$ & 13 & $2 \cdot 55^{*}$ & $1 \cdot 08-9 \cdot 64$ & 12 & $1 \cdot 46$ & $0 \cdot 30-5 \cdot 30$ \\
\hline Cells $\times 10^{-6} / \mathrm{ml}$ & 13 & $1 \cdot 35^{*}$ & $0 \cdot 76-8 \cdot 62$ & 12 & $0 \cdot 87$ & $0 \cdot 30-2 \cdot 65$ \\
\hline
\end{tabular}

*P $<0.05$, compared with controls.

\section{Results}

At laparoscopy, 13 patients were found to have endometriosis (Score 1, N =9; Score 2, $N=4$; American Fertility Society, 1979); 12 patients without endometriosis served as controls. Based on the oestradiol-17 $\beta$ and progesterone concentrations in peritoneal fluid and serum (Bouckaert et al., 1986), 5 patients in the endometriosis group were classified as being in the follicular and 8 as being in the luteal phase of the cycle. The figures for controls were 2 and 10 respectively. Values of C-reactive protein were below the level of detection in all peritoneal fluid and serum specimens.

As shown in Table 1, the median value of the total cell number and the median value of the cell number per ml peritoneal fluid in endometriosis patients were higher than in controls, both differences were statistically significant $(P<0.05)$.

The viability of the cells and the percentage of macrophages in the differential count were not significantly different in the two groups (Table 2). Table 3 shows the results of the sheep RBC 
Table 2. Viability and percentage of macrophages in peritoneal fluid

\begin{tabular}{lcccccccc}
\hline & \multicolumn{3}{c}{ Endometriosis samples } & & \multicolumn{3}{c}{ Control samples } \\
\cline { 2 - 3 } \cline { 8 - 9 } & $n$ & Median & Range & & $n$ & Median & Range \\
\hline Viability (\%) & 13 & $82 \cdot 0^{*}$ & $31-99$ & & 12 & $80 \cdot 0$ & $42-97$ \\
Macrophages (\%) & 13 & $93 \cdot 0^{*}$ & $92-98$ & & 12 & $91 \cdot 0$ & $27-97$ \\
\hline
\end{tabular}

${ }^{*} P>0.05$, compared with controls.

Table 3. Phagocytosis of sheep RBCs and chemiluminescence by peritoneal fluid macrophages

\begin{tabular}{lcccccccc}
\hline & \multicolumn{3}{c}{ Endometriosis samples } & & \multicolumn{3}{c}{ Control samples } \\
\cline { 2 - 3 } \cline { 7 - 8 } & $n$ & Median & Range & & $n$ & Median & Range \\
\hline $\begin{array}{l}\text { Sheep RBCs (\%) } \\
\begin{array}{l}\text { Chemiluminescence } \\
\text { (units) }\end{array}\end{array}$ & 13 & $90 \cdot 0^{* *}$ & $12-97$ & 12 & $35 \cdot 5$ & $3-76$ \\
\hline
\end{tabular}

${ }^{*} P<0.001 ;{ }^{* *} P<0.0001$ compared with controls.

phagocytosis and of the chemiluminescence of peritoneal cells in endometriosis and controls. A statistically significant difference in sheep RBC phagocytosis existed between endometriosis patients and controls $(P<0.0001)$. The lower level of chemiluminescence in endometriosis patients also was statistically significant $(P<0.001)$.

\section{Discussion}

Increased total peritoneal fluid cell numbers and cell concentrations, with a percentage of macrophages of $80 \%$ and higher have repeatedly been reported in the literature in patients with endometriosis as compared to controls, although the differences were not always statistically significant (Haney et al., 1981; Badawy et al., 1984; Halme et al., 1982, 1984; Olive et al., 1985). In the present study we confirmed the increased number and the increased concentration of peritoneal cells in patients with endometriosis as compared to controls. The peritoneal cavity is normally populated by resident macrophages. Secondary to several stimuli, including shedding of endometrial tissue in addition to ovulation and retrograde menstruation, monocytes are attracted from the bloodstream and convert to differentiated macrophages, leading to an increased concentration of peritoneal cells. An acute inflammatory process will change the peritoneal cell number considerably. The finding in our study that C-reactive protein was below the level of detection in peritoneal fluid and in serum in patients with endometriosis and in the control group indicates that there was no acute intraperitoneal inflammatory process in either group at the time of the laparoscopy (Lehtinen et al., 1986).

The phagocytic capacity of peritoneal macrophages measured by the uptake of opsonized zymosan has been reported to be comparable in endometriosis patients and controls (Halme et al., 1984). We found an increased phagocytosis of sheep RBCs by macrophages recovered from the peritoneal fluid of patients with endometriosis as compared to controls. Viability and percentage of macrophages in peritoneal cells were the same in each group (Table 2). The increased ability of peritoneal macrophages to phagocytose antibody-coated particles (sheep RBCs) supports the findings of London et al. (1985) concerning macrophage-mediated sperm killing as a possible cause of subfertility. 
A remarkable finding of the present investigation was the significantly reduced chemiluminescence measured in peritoneal macrophages of endometriosis patients. The dissociation that we found between an increased phagocytosis and a reduced chemiluminescence of macrophages in endometriosis patients compared with controls may be due to different degrees of differentiation of the peritoneal macrophages. After stimulation macrophages undergo changes in metabolism and differentiate in a stepwise fashion (Cohn, 1978; Yamamoto \& Johnston, 1984; Johnson et al., 1986). Following this concept, the levels of phagocytosis and chemiluminescence measured in control patients represent a baseline steady state level while the enhanced phagocytosis and the diminished chemiluminescence in endometriosis patients indicate the next step in differentiation, with the macrophages being engaged in phagocytosis but having left the stage of $\mathrm{O}_{2}$ production.

The concept of a more advanced stage of differentiation of peritoneal macrophages in patients with endometriosis is supported by two reports. Fakih et al. (1987) reported on the presence of interleukin-1, a protein produced by differentiated peritoneal macrophages and a mediator of host responses, in the peritoneal fluid of patients with endometriosis and not in that of controls without the disease. Halme et al. (1987), extending earlier observations (Halme et al., 1984), described the existence of larger, more mature macrophages in the peritoneal fluid of patients with endometriosis.

In conclusion, we found increased sheep RBC phagocytosis and a decreased level of chemiluminescence of peritoneal macrophages in patients with endometriosis compared to controls. This is consistent with an advanced level of differentiation of peritoneal macrophages in patients with endometriosis. These macrophages may interfere with gametes and pre-implantation embryos and thus contribute to endometriosis-associated subfertility.

\section{References}

American Fertility Society (1979) Classification of endometriosis. Fert. Steril. 32, 633-634.

Badawy, S.Z.A., Cuenca, V., Marshall, L., Munchback, R., Rinas, A.C. \& Coble, D.A. (1984) Cellular components in peritoneal fluid in infertile patients with and without endometriosis. Fert. Steril. 42, 704-708.

Bouckaert, P.X.J.M., Evers, J.L.H., Doesburg, W.H., Schellekens, L.A. \& Rolland, R. (1986) Patterns of changes in glycoproteins, polypeptides, and steroids in the peritoneal fluid of women during the periovulatory phase of the menstrual cycle. J. clin. Endocr. Metab. 62, 293-299.

Cohn, Z.A. (1978) The activation of mononuclear phagocytes: fact, fancy and future. J. Immunol. 121, 813-816.

Fakih, H., Baggett, B., Holtz, G., Tsang, K-Y., Lee, J.C. \& Williamson, H.O. (1987) Interleukin-1: a possible role in the infertility associated with endometriosis. Fert. Steril. 47, 213-217.

Halme, J., Becker, S., Hammond, M.G. \& Raj, S. (1982) Pelvic macrophages in normal and infertile women: the role of patent tubes. Am. J. Obstet. Gynecol. 142, 890-895.

Halme, J., Becker, S. \& Wing, R. (1984) Accentuated cyclic activation of peritoneal macrophages in patients with endometriosis. Am. J. Obstet. Gynecol. 148, $85-90$.

Halme, J., Becker, S. \& Haskill, S. (1987) Altered maturation and function of peritoneal macrophages: possible role in pathogenesis of endometriosis. Am. J. Obstet. Gynecol. 156, 783-789.

Haney, A.F., Muscato, J.J. \& Weinberg, J.B. (1981) Peritoneal fluid cell populations in infertility patients. Fert. Steril. 35, 696698 .
Hendrix, M.G.R., Bruggeman, C.A. \& Van Boven, C.P.A. (1986) Alterations of the functional state of peritoneal macrophages during rat cytomegalovirus infection in vitro. FEMS Lett. 33, 111-115.

Johnson, W.J., DiMartino, M.J. \& Hanna, N. (1986) Macrophage activation in rat models of inflammation and arthritis: determination of markers of stages of activation. Cell. Immunol. 103, 54-64.

Lehtinen, M., Laine, S., Heinonen, P.K., Teisala, K., Miettinen, A., Aine, R., Punnonen, R., Grönroos, P. \& Paavonen, J. (1986) Serum C-reactive protein determination in acute pelvic inflammatory disease. $\mathrm{Am}$.J. Obstet. Gynecol. 154, 158-160.

London, S.N., Haney, A.F. \& Weinberg, J.B. (1985) Macrophages and infertility: enhancement of human macrophage-mediated sperm killing by antisperm antibodies. Fert. Steril. 43, $274-278$.

Mancini, G., Carbonara, A.O. \& Heremans, J.F. (1965) Immunochemical quantitation of antigens by single radial immunodiffusion. Immunochemistry 2 , $235-254$.

Muscato, J.J., Haney, A.F. \& Weinberg, J.B. (1982) Sperm phagocytosis by human peritoneal macrophages: a possible cause of infertility in endometriosis. Am. J. Obstet. Gynecol. 144, 503-510.

Olive, D.L., Weinberg, J.B. \& Haney, A.F. (1985) Peritoneal macrophages and infertility: the association between cell number and pelvic pathology. Fert. Steril. 44, 772-777.

Yamamoto, K. \& Johnston, R.B., Jr (1984) Dissociation of phagocytosis from stimulation of the oxidative metabolic burst in macrophages. J.exp. Med.159,405-416.

Received 24 July 1987 